\title{
Uso da própolis no controle "in vitro" do fungo Colletotrichum gloeosporioides causador da antracnose em berinjela
}

\author{
Rubens Felippe Pastana ${ }^{1}$, Gustavo Haralampidou Costa Vieira ${ }^{1}$, Patricia Pereira Machado ${ }^{1}$ \\ ${ }^{1}$ Universidade Estadual de Mato Grosso do Sul - UEMS, Unidade Universitária de Cassilândia, Mato Grosso do Sul, Brasil. E-mail: \\ rubinho_63@hotmail.com,gcv@uems.br, paty.pereira.pba@hotmail.com
}

Recebido: 10/10/2015; Aceito: 21/12/2015.

\section{RESUMO}

O trabalho foi desenvolvido com o objetivo de determinar o efeito da própolis no controle "in vitro" do fungo Colletotrichum gloeosporioides. A atividade antifúngica da própolis foi determinada através do desenvolvimento do fungo em meio de cultura BDA (batata-dextrose-ágar) acrescido da referida substância em diferentes concentrações, conforme os seguintes tratamentos: 1) testemunha absoluta (sem aplicação); 2) testemunha com aplicação de $4 \mathrm{~mL} \mathrm{~L}^{-1}$ de fungicida (Cupravit Azul BR - oxicloreto de cobre inorgânico); 3) $4 \mathrm{~mL} \mathrm{~L}^{-1}$ de própolis; 4) $8 \mathrm{~mL} \mathrm{~L}^{-1}$ de própolis; 5) $16 \mathrm{~mL} \mathrm{~L}^{-1}$ de própolis; e, 6) $32 \mathrm{~mL} \mathrm{~L}^{-1}$ de própolis. O crescimento micelial do fungo foram avaliados após 48, 72 e $96 \mathrm{~h}$ de inoculação. Os resultados indicaram que o uso de própolis na maior concentração $\left(32 \mathrm{~mL} \mathrm{~L}^{-1}\right)$ foi eficiência no controle do crescimento micelial "in vitro" do fungo Colletotrichum gloeosporioides.

Palavras-chave: controle alternativo, Apis mellifera, inibição do crescimento micelial.

\section{Use of propolis on "in vitro" control of the fungus Colletotrichum gloeosporioides causing anthracnose in eggplant}

\begin{abstract}
Use of natural products is an alternative strategy to control postharvest fungal rotting of fruit and vegetables. This study was conducted with the objective of determine the effect of propolis on "in vitro" control of the fungus Colletotrichum gloeosporioides. The antifungal activity of propolis was determined by fungal growth on PDA (potato-dextrose-agar) culture medium plus of this substance in different concentrations, according to the following treatments: 1) control treatment (without application); 2) control with application $4 \mathrm{~mL} \mathrm{~L}^{-1}$ of fungicide (Cupravit BR Blue - inorganic copper oxychloride); 3) $4 \mathrm{~mL} \mathrm{~L}^{-1}$ of propolis; 4) $8 \mathrm{~mL} \mathrm{~L}^{-1}$ of propolis; 5) $16 \mathrm{~mL} \mathrm{~L}^{-1}$ of propolis; and, 6) $32 \mathrm{~mL} \mathrm{~L}^{-1}$ of propolis. The fungus mycelial growth was evaluated after 48, 72 and 96 hours of inoculation. The results indicated that the use of propolis in higher concentration $\left(32 \mathrm{~mL} \mathrm{~L}^{-1}\right)$ was efficient in the "in vitro" control of mycelial growth of the fungus Colletotrichum gloeosporioides.
\end{abstract}

Key words: alternative control, Apis mellifera, mycelial growth inhibition. 


\section{Introdução}

A berinjela (Solanum melongena L.) é uma planta perene, porém cultivada como cultura anual, pertencente à família Solanaceae. Esta planta inicialmente foi cultivada nas regiões tropicais do Oriente e, há séculos, vêm sendo cultivado por árabes e chineses (FILGUEIRA, 2003). Atualmente, o Brasil destina pouco mais de 1500 ha para o cultivo da berinjela. No entanto, há uma tendência para o aumento do plantio no país tendo em vista a riqueza nutricional e propriedades medicinais da berinjela que têm favorecido o consumo desta olerícola; o alimento é cada vez mais presente na mesa dos brasileiros (OLIVEIRA et al., 2009).

A berinjela é uma hortaliça termófila necessitando de temperaturas elevadas para seu desenvolvimento reprodutivo e vegetativo (SOUSA et al., 1997). Estas condições associadas a elevados índices de umidade favorecem o desenvolvimento de patógenos, tais como fungos do gênero Colletotrichum, parasita comum a muitas angiospermas (ALEXOPOULOS, 1996).

O fungo Colletotrichum gloeosporioides é o agente etiológico da antracnose em hortaliças, considerada uma das principais doenças do pimentão (Capsicum annuum L.), jiló (Solanum gilo Raddi) e berinjela (Solanum melongena L.). Os maiores índices de incidência desta doença ocorrem no período pós-colheita devido a um controle inadequado, resultando em grandes prejuízos econômicos. Em alguns casos observam-se perdas de até $100 \%$ na produção (SERRA; SILVA, 2004).

O patógeno ataca todos os órgãos da parte aérea da planta, mas somente os frutos exibem sintomas típicos que se manifestam em forma de depressão circular de diâmetro variável. Em ambientes úmidos aparecem cirros na parte deprimida, que são massas de conídios de cor rosada produzidos em acérvulos (KUROZAWA; PAVAN, 1997). Esses sintomas por vezes aparecem após a colheita, durante o transporte e a comercialização dos frutos, acarretando em prejuízos ainda maiores. A principal forma de controle deste fungo é realizada com o uso de fungicidas que, muitas vezes, deixam resíduos no produto.

Como a berinjela é consumida quase in natura, na maioria das vezes, seria ideal o desenvolvimento de estudos que garantissem a produção deste alimento isento de agrotóxicos, visando a qualidade do produto que chega ao consumidor. Neste contexto, a própolis vem ganhando destaque por apresentar características antimicrobianas e antifúngicas, dentre outras, que muitas vezes inibem o desenvolvimento desses patógenos (PEREIRA et al., 2002).

Considerando as características climáticas do Estado de Mato Grosso do Sul que favorecem o cultivo da berinjela, associada à crescente demanda pelo aumento da oferta de hortaliças de qualidade, o presente trabalho foi desenvolvido com o objetivo de determinar a eficiência da própolis no controle "in vitro" do fungo $C$. gloeosporioides causador da antracnose nos frutos da berinjela, possibilitando, assim, uma alternativa de controle isenta de substâncias tóxicas garantindo a qualidade dos frutos após a colheita

\section{Material e Métodos}

O trabalho foi conduzido no laboratório de Fitossanidade da Universidade Estadual do Mato Grosso do Sul, município de Cassilândia/MS (1906'48" S; $51^{\circ} 44^{\prime} 03$ ” W), no período de agosto de 2012 a julho de 2013.

A própolis foi produzida em colmeias de Apis melífera, a extração ocorreu com auxílio de coletores de própolis inteligente (CPI), instalados em dez colmeias de abelhas africanizadas. O produto colhido foi diluído em álcool de cereais na concentração de $30 \%$, conforme a metodologia proposta por Adomar (1996).

Os fungos foram isolados diretamente dos frutos com sintomas e inoculados em meio de cultura BDA (batata-dextrose-ágar) mantidos em BOD à temperatura de $27^{\circ} \mathrm{C}$ e fotoperíodo de 12 horas.

A atividade antifúngica da própolis foi determinada através do desenvolvimento colonial do fungo em meio de cultura com as substâncias correspondentes. Para tanto, discos de $5 \mathrm{~mm}$ de diâmetro com propágulos do fungo foram transferidos para placas de petri contendo a própolis em diferentes concentrações incorporadas ao meio BDA. As placas foram incubadas em BOD a 27 ${ }^{\circ} \mathrm{C}$, com fotoperíodo de 12 horas.

$\mathrm{O}$ delineamento experimental utilizado foi inteiramente casualizado com 6 tratamentos: 1) testemunha absoluta (sem aplicação); 2) testemunha com aplicação de $4 \mathrm{~mL} \mathrm{~L}^{-1}$ de fungicida Cupravit Azul BR (oxicloreto de cobre inorgânico); 3) $4 \mathrm{~mL} \mathrm{~L}^{-1} \mathrm{de}$ própolis; 4) $8 \mathrm{~mL} \mathrm{~L}^{-1}$ de própolis; 5) $16 \mathrm{~mL} \mathrm{~L}^{-1} \mathrm{de}$ própolis; e, 6) $32 \mathrm{~mL} \mathrm{~L}^{-1}$ de própolis, e cinco repetições. Os tratamentos 1 e 2 foram constituídos de duas testemunhas para comparação das médias, sendo uma positiva (+) composta apenas pelo meio BDA e a outra negativa $(-)$, composta por BDA mais fungicida. $O$ fungicida utilizado está registrado no Agrofit (Sistema de Agrotóxicos Fitossanitários) do Ministério da Agricultura.

As avaliações do desenvolvimento colonial foram realizadas após 48, 72 e 96 h de inoculação do fungo. Para tanto, foram obtidos os diâmetros das colônias (média de duas medidas diametralmente opostas) com auxílio de paquímetro analógico.

Os dados foram submetidos à análise de variância e as médias comparadas pelo teste de Tukey a $5 \%$ de probabilidade. A análise de regressão foi usada para estabelecer a relação entre as concentrações de própolis e os valores de crescimento micelial. 


\section{Resultados e Discussão}

A própolis influenciou o crescimento micelial do fungo Colletotrichum gloesporioides, observando-se a relação dose dependente entre as concentrações usadas e o desenvolvimento do fungo, observando-se a diminuição do seu desenvolvimento com o aumento das doses, exceto apenas para as doses de 4 e $8 \mathrm{~mL} \mathrm{~L}^{-1}$ no último período de observação (Tabela 1, Figura 1). Por outro lado, apenas os resultados obtidos com a maior dose testada (32 $\left.\mathrm{mL} \mathrm{L}^{-1}\right)$ no último período de avaliação foi semelhante aos resultados observados para o fungicida (Tabela 1).

Substância utilizada há vários séculos em diversas partes do mundo, a própolis é conhecida por suas propriedades antimicrobianas (MARCUCCI et al., 2000). Possui uma complexa composição química e suas atividades biológicas são atribuídas a resinas vegetais. (SALATINO et al., 2005).

Tabela 1. Eficiência de diferentes concentrações de própolis no controle do fungo Colletrotrichum gloeosporioides. Cassilândia (MS), 2013

\begin{tabular}{|c|c|c|c|c|}
\hline \multirow{2}{*}{\multicolumn{2}{|c|}{ Tratamento }} & \multicolumn{3}{|c|}{ Crescimento micelial $(\mathrm{cm})$} \\
\hline & & $48 \mathrm{~h}$ & $72 \mathrm{~h}$ & $96 \mathrm{~h}$ \\
\hline & Testemunha (sem aplicação) & $2,56 \mathrm{a}$ & $3,99 \mathrm{a}$ & $5,32 \mathrm{a}$ \\
\hline & Fungicida (4 ml L' $\left.{ }^{-1}\right)$ & $0,00 \mathrm{e}$ & $0,00 \mathrm{e}$ & $0,00 \mathrm{c}$ \\
\hline & $4 \mathrm{ml} \mathrm{L}^{-1}$ de propolis & $2,10 \mathrm{ab}$ & $2,96 \mathrm{~b}$ & $4,03 \mathrm{ab}$ \\
\hline & $8 \mathrm{ml} \mathrm{L}^{-1}$ de propolis & $2,62 \mathrm{bc}$ & $2,58 \mathrm{~b}$ & $4,36 \mathrm{~b}$ \\
\hline & $16 \mathrm{ml} \mathrm{L}^{-1}$ de propolis & $1,39 \mathrm{c}$ & $2,11 \mathrm{c}$ & $2,90 \mathrm{~b}$ \\
\hline & $32 \mathrm{ml} \mathrm{L}^{-1}$ de propolis & $0,66 \mathrm{~d}$ & $0,95 \mathrm{~d}$ & $1,18 \mathrm{c}$ \\
\hline \multicolumn{2}{|l|}{ Teste F } & 58,41 & 192,80 & 36,25 \\
\hline \multicolumn{2}{|l|}{$\mathrm{CV}(\%)$} & 19,75 & 11,00 & 25,43 \\
\hline
\end{tabular}

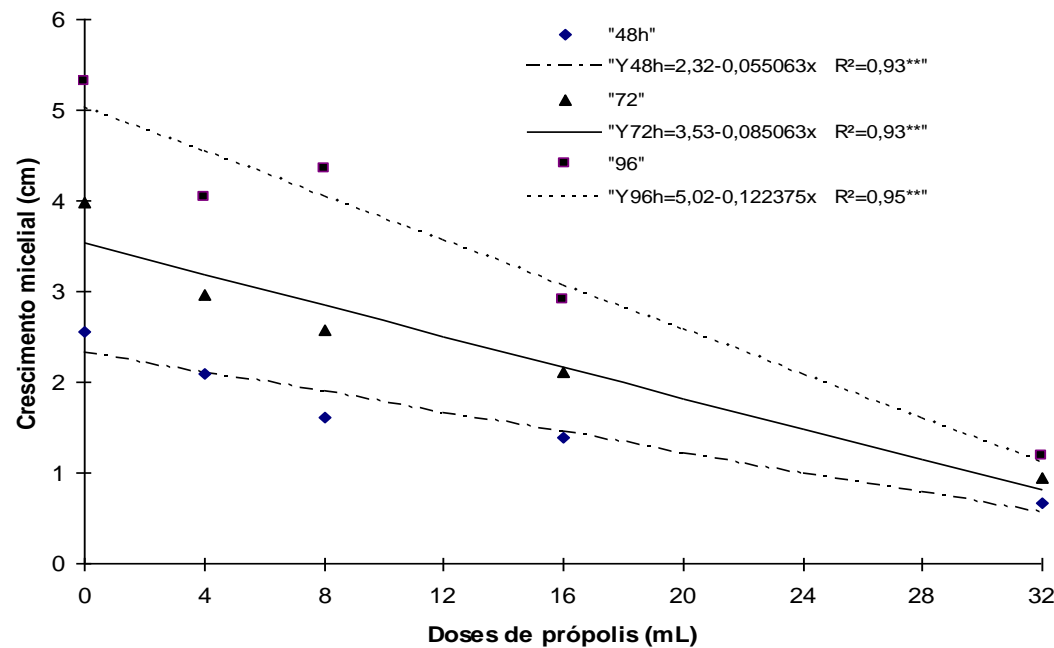

Figura 1. Crescimento micelial do fungo C. gloesporioides em relação às diferentes concentrações de própolis. Cassilândia (MS), 2013.

Dentre os compostos de origem vegetal pode-se citar os flavonóides, ácidos, esteres e aldeidos fenólicos (CASTALDO; CAPASSO, 2002), sendo atribuído aos flavonóides propriedades fungicidas e fungistáticas (FONTANA et al., 2004) e em menor escala a associação desses com ácidos aromáticos fenólicos e seus derivados (BURDOK, 1998).

Embora este trabalho não tenha caracterizado através de análises físico-químicas a própolis estudada, pode-se supor que os resultados observados são decorrentes da ação dos flavonóides pois sabe-se que independente da origem, essa substância sempre apresenta tal composto que lhe confere ação antimicrobiana, sendo esta a função primordial para a manutenção da vida na colméia (BANKOVA et al., 1998).

Outros estudos que apresentaram resultados semelhantes aos encontrados neste trabalho foram realizados por Monzote et al. (2012) e Maekawa et al. (2013). Monzote et al. (2012) determinaram a eficiência 
do extrato de própolis no controle do fungo Trichophyton rubrum. Maekawa et al. (2013) determinaram o efeito do extrato glicólico da própolis nos fungos Candida albicans e Enterococcus faecalis, observando que a própolis possui efeito inibitório sobre esses microrganismos.

Estes estudos demonstram irrefutavelmente as propriedades antifúngicas da própolis, fazendo dessa substância uma alternativa importante ao emprego nos programas de manejo integrado de pragas e sistemas sustentáveis de produção orgânica.

\section{Conclusões}

O uso de própolis a partir da concentração de $8 \mathrm{~mL}$ $\mathrm{L}^{-1}$ possui uma relação inversamente proporcional ao crescimento micelial do fungo Colletotrichum gloesporioides em condições in vitro.

\section{Referências Bibliográficas}

ADOMAR, J. Informações pessoais. Cooperado da CONAP (Cooperativa Nacional de Apitoxina), Belo Horizonte: Minas Gerais, 1996.

ALEXOPOULOS, C.J.; MIMS, C.W.; BLACKWELL, M. Introductory mycology. 4. ed. New York: John Wiley, 1996. 868 p.

BANKOVA, V.; KRASKEVA, G. B.; POPOV, S.; SFORCIN, J. M.; FUNARI, S. R. C. Seasonal variations of the chemical composition of Brazilian propolis. Apidologie, $\mathrm{v}$. 29, n. 1, p. 361-367, 1998 .

BURDOCK, G. A. Review of the biological properties and toxicity of bee propolis. Food and Chemical Toxicology, v. 36, n. 4, p. 347- 363, 1998.

CASTALDO, S.; CAPASSO, F. Propolis, an old remedy used in modern medicine. Fitoterapia, v. 73, n. 1, p. 1-6, 2002.

FONTANA, J. D.; ADELMANN, J.; PASSOS, M.; MARASCHIN, M.; LACERDA, C. A.; LANÇAS, F. M. Propolis: chemical micro-heterogeneity and bioactivity. New Jersey: Humana press, p. $203-218,2004$.
FILGUEIRA, F. A. R. Solanáceas: agrotecnologia moderna na produção de tomate, batata, pimentão, pimenta, berinjela, e jiló. Lavras: UFLA, 2003. 333 p.

KUROZAWA, C.; PAVAN, M.A. Doenças das solanáceas. In: KIMATI, H.; AMORIM, L.; BERGAMIN FILHO, A.; CAMARGO, L.E.A.; REZENDE, J.A.M. (Eds.) Manual de Fitopatologia: Doenças das plantas cultivadas, v. 2, p.621, 1997.

MAEKAWA, L.E.; VALERA, M.C.; OLIVEIRA, L.D.; CARVALHO, C.A.T.; CAMARGO, C.H.R.; JORGE, A.O.C. Effect the Zingiber officinale and propolis on microorganism and endotoxins in root canals. Journal of Apllicied Oral Science, v. 21, n. 1, p. 25-31, 2013.

MARCUCCI, M.C.; FERRERES, F.; CUSTODIO, A.R.; FERREIRA, M.M.; BANKOVA, V. S.; GARCIA-VIGUERA, C.; BRETZ, W.A. Evaluation of phenolic compounds in Brazilian propolis from different geographic regions. Zeitschrift fur Naturforschungv, v. 55, n. 1, p. 76-81, 2000.

MONZOTE, L.; CUESTA-RUBIO, O.; FERNANDEZ, M. C.; Hernandez. I. M.; Fraga, J.; Pérez, K.; Kerstens, M.; Maes, L.; Cos, P. In vitro antimicrobial assessment of Cuban propolis extracts. Memórias do Instituto Oswaldo Cruz. v. 107, n. 8, p. 978-984, 2012.

OLIVEIRA, A. B.; HERNANDEZ, F. F. F.; ASSIS JÚNIOR, R. N. Absorção de nutrientes em mudas de berinjela cultivadas em pó de coco verde. Revista Caatinga, v. 22, n. 02, p. 139 143, 2009.

PEREIRA, A. dos S; SEIXAS, F. R. M. S; NETO, RADLER, $\mathrm{N}$ de A. Própolis: 100 anos de pesquisa e suas perspectivas futuras. Química Nova, v. 25, n. 2, p. 321-326, mar./abr. 2002.

SALANTINO, A.; TEIXEIRA, E. W.; NEGRI, G.; MESSAGE, D. Origin and chemical variation of Brazilian propolis. Evidence-Based Complementary and Alternative Medicine, v. 2, n. 1, p. 33-38, 2005.

SERRA, I. M. R. de S.; SILVA, G. S. da. Caracterização Morfofisiológica de Isolados de Colletotrichum gloeosporioides Agentes de Antracnose em Frutíferas no Maranhão. Summa Phytopathologica, v. 30, n. 4, p. 475-480, 2004.

SOUSA, J. A.; LEDO, F. J. S.; SILVA, M. R. Produção de mudas de hortaliças em recipientes. Rio Branco: EmbrapaCPAF/AC, 1997. 19 p. (Circular Técnica, 19). 